urothelial carcinoma. There are a variety of reasons for false-negative diagnoses of ureteral and bladder cancer during MDCT- urography. Early-enhanced thin-section MDCT of a full bladder with urine can decrease the number of false negatives bladder studies. This "bladder -wall phase ", obtained 60 seconds after contrast injection has superior accuracy for detection of small lesions in comparison with the excretory phase alone (bladder fully distended by opacified urine), as used by the authors. However small flat tumors that do not appear as filling defects and carcinoma in situ tumors, are almost impossible to be detected by MDCT-urography. For this reason, although not a perfect test, cystocopy remains the reference standard procedure in the investigation of hematuria.

Dr. Adilson Prando

Head, Department of Radiology and

Diagnostic Imaging, Vera Cruz Hospital

Campinas, São Paulo, Brazil

E-mail: adilson.prando@gmail.com

doi: 10.1590/S1677-55382010000500019

\title{
Prostate cancer managed with active surveillance: role of anatomic MR imaging and MR spectroscopic imaging
}

Fradet V, Kurhanewicz J, Cowan JE, Karl A, Coakley FV, Shinohara K, Carroll PR

Department of Urology, Helen Diller Family Comprehensive Cancer Center, University of California, San Francisco, San Francisco, California, USA

Radiology. 2010; 256: 176-83

Purpose: To determine the role that magnetic resonance (MR) imaging and MR spectroscopic imaging findings obtained at the time of diagnosis play in the progression of disease in patients whose prostate cancer is being managed with active surveillance and to compare the role of these findings with the role of transrectal ultrasonography (US) findings.

Materials and Methods: The institutional review board approved this HIPAA-compliant retrospective study, and informed consent was obtained from all patients whose records were to be entered into the research database. All patients who had prostate cancer managed with active surveillance and who had undergone both MR imaging and MR spectroscopic imaging of the prostate and transrectal US at time of diagnosis were identified. Two urologists blinded to the clinical outcome in these patients independently reviewed and dichotomized the MR imaging report and the MR spectroscopic imaging report as normal or suggestive of malignancy. One experienced urologist performed all US examinations that were then dichotomized similarly. Uni- and multivariate (with use of standard clinical variables) Cox models were fitted to assess time to cancer progression, defined as Gleason score upgrading, prostate-specific antigen velocity of more than 0.75 (microg x L(-1))/y, or initiation of treatment more than 6 months after diagnosis.

Results: The final cohort included 114 patients with a median follow-up of 59 months. Patients with a lesion that was suggestive of cancer at MR imaging had a greater risk of the Gleason score being upgraded at subsequent biopsy (hazard ratio, 4.0; 95\% confidence interval: 1.1, 14.9) than did patients without such a lesion. Neither MR spectroscopic imaging nor transrectal US could be used to predict cancer progression.

Conclusion: Abnormal prostate MR imaging results suggestive of cancer may confer an increased risk of Gleason score upgrade at subsequent biopsy. Although expensive, prostate MR imaging may help in counseling potential candidates about active surveillance. 


\section{Editorial Comment}

The incorporation of combined MRI/MRSI and multiparametric MRI in the active surveillance program is still investigational. The possible application of these techniques is based on the fact that volumetric and metabolic data correlates with tumor aggressiveness (1).

Recently study has been shown that combined MR imaging and MR spectroscopic imaging findings and Ki-67 (a proliferation marker), pAkt (a serine-threonine kinase), and androgen receptor values correlated with each other and with clinically insignificant and significant prostate cancers (2). In another study, MRI/MRSI models performed better than the clinical models for predicting the probability of insignificant prostate cancer (3). In the study by Fradet et al., patients presenting a hypointense focal area on T2-weighted image, suspicious for cancer at the time of diagnosis, had a greater risk of the Gleason score being upgraded at subsequent biopsy than did patients without such a lesion. Interesting is that MR spectroscopic imaging which is more specific for tumor characterization, than conventional T2-weighted images, was not useful to predict cancer progression. We agree with the authors that after appropriate validation, the MRI and MRI/MRSI models might help in counseling patients who are considering choosing deferred therapy.

\section{References}

1. Zakian KL, Sircar K, Hricak H, Chen HN, Shukla-Dave A, Eberhardt S, et al.: Correlation of proton MR spectroscopic imaging with gleason score based on step-section pathologic analysis after radical prostatectomy. Radiology. 2005; 234: 804-14.

2. Shukla-Dave A, Hricak H, Ishill NM, Moskowitz CS, Drobnjak M, Reuter VE, et al.: Correlation of MR imaging and MR spectroscopic imaging findings with Ki-67, phospho-Akt, and androgen receptor expression in prostate cancer. Radiology. 2009; 250: 803-12.

3. Shukla-Dave A, Hricak H, Kattan MW, Pucar D, Kuroiwa K, Chen HN, et al.: The utility of magnetic resonance imaging and spectroscopy for predicting insignificant prostate cancer: an initial analysis. BJU Int. 2007; 99: 786-93.

Dr. Adilson Prando

Head, Department of Radiology and

Diagnostic Imaging, Vera Cruz Hospital

Campinas, São Paulo, Brazil

E-mail: adilson.prando@gmail.com

\section{PATHOLOGY}

doi: 10.1590/S1677-55382010000500020

\section{Optimizing performance and interpretation of prostate biopsy: a critical analysis of the literature}

Chun FK, Epstein JI, Ficarra V, Freedland SJ, Montironi R, Montorsi F, Shariat SF, Schröder FH, Scattoni V Department of Urology, University Hospital Hamburg-Eppendorf, University of Hamburg, Hamburg, Germany

Eur Urol. 2010 Sep 4. [Epub ahead of print]

Context: The number and location of biopsy cores and the interpretation of prostate biopsy in different clinical settings remain the subjects of continuing debate.

Objective: Our aim was to review the current evidence regarding the performance and interpretation of initial, repeat, and saturation prostatic biopsy. 